\title{
Filigrane
}

Écoutes psychothérapiques

\section{Au-delà du mal... le non-sujet. Entretien avec Michel Brun}

\section{Michel Peterson}

Volume 23, numéro 1, printemps 2014

URI : https://id.erudit.org/iderudit/1026082ar

DOI : https://doi.org/10.7202/1026082ar

Aller au sommaire du numéro

Éditeur(s)

Revue Santé mentale au Québec

ISSN

1192-1412 (imprimé)

1911-4656 (numérique)

Découvrir la revue

Citer ce document

Peterson, M. (2014). Au-delà du mal... le non-sujet. Entretien avec Michel Brun. Filigrane, 23(1), 139-148. https://doi.org/10.7202/1026082ar d'utilisation que vous pouvez consulter en ligne.

https://apropos.erudit.org/fr/usagers/politique-dutilisation/ 


\title{
Au-delà du mal... le non-sujet. Entretien avec Michel Brun ${ }^{1}$
}

\author{
Michel Peterson
}

Michel Peterson: Michel Brun, vous êtes philosophe et psychanalyste, vous avez étudié à Dakar et à Lille et vous avez participé durant de nombreuses années aux séminaires de Jean-Yves Tatte et du $\mathrm{D}^{\mathrm{r}}$ Stoianoff-Nenof puis à ceux de la Cause freudienne. Vous pratiquez la psychanalyse et vous enseignez le yoga. Vous vous intéressez d'ailleurs depuis plus de trente ans aux différents aspects de la pensée orientale, auxquels vous avez consacré différents articles. À la lecture de vos travaux, on s'aperçoit que pour vous - comme pour des cliniciens et auteurs comme Christiane Berthelet-Lorelle et Pierre Pelletier —, la psychanalyse peut partager avec le yoga un enjeu majeur, à savoir le désencombrement de soi.

Je voudrais ouvrir notre entretien en partant du second chapitre de votre livre, Dieu, encore? Jalons pour une théologie négative contemporaine ${ }^{2}$, où il est question de l'expression du mal parmi les humains, qui s'inscrit en contrepoint du désencombrement que j'évoquais à l'instant. Il s'agit à mon sens d'une question absolument fondamentale, qui m'a rappelé plusieurs travaux écrits dans un horizon très différent. Je pense par exemple à ceux de Hannah Arendt sur la banalisation du mal, de Pierre Fédida sur le déshumain, ainsi qu'aux œuvres de plusieurs grands poètes, de plusieurs grands écrivains.

Deux moments en particulier me paraissent essentiels dans ce chapitre. Vous dégagez le premier ainsi: «Le mal est une réalité permanente devant laquelle la raison défaille. » À propos de cette affirmation tout de même troublante, ma question est la suivante: le mal est-il un dérapage par rapport à la Raison ou, dans certaines situations, le fait d'une décision rationnelle? Je vais lire une deuxième phrase parce qu'elles sont peut-être liées, mais nous pourrons déployer cela comme vous le souhaitez. Le deuxième moment se traduit par l'énoncé suivant: «En suivant le fil rouge de la souffrance de l'humanité, nous attendons toujours, mais en vain, que Dieu s'explique sur Auschwitz, et ce d'autant plus qu'un certain nombre de bourreaux nazis, certes âgés, coulent encore des jours heureux en des lieux protégés... » (2012, 
64). Il s'agit d'un moment extrêmement fort de cette partie de votre livre, qui concerne ce que vous appelez « les figures ambiguës du divin ». J'aimerais vous entendre sur le lien entre la Raison, Dieu et la dimension génocidaire de l'humanité.

Michel Brun : La rationalisation du mal évoque pour moi la programmation du génocide. Pensons à Auschwitz et à l'ensemble des camps de concentration. Il y eut une véritable programmation de la mise à mort, une rationalisation des moyens par lesquels nous allions éliminer cet autre qui, en fait, nous est très proche. Ce qui me frappe, c'est que celui qu'on essaie d'éliminer de façon délibérée, rationnelle, cohérente, systématique parfois, n’est pas quelqu'un qui se situe dans un horizon très lointain, mais celui qui nous est très proche. Ça commence par ça. Un exemple récent est celui du Rwanda: les ennemis étaient pratiquement des frères. De même, en Yougoslavie, le massacre des Bosniaques a été perpétré par ceux qui étaient très proches, c'est-à-dire des Serbes; et au Cambodge, c'est le peuple qui fut assassiné.

MP: Comme ce fut également le cas au Goulag.

MB : Au Goulag, effectivement. Je me demande — c'est un peu le psychanalyste qui parle - si ça ne nous ramène pas à la problématique de la rivalité imaginaire qui ne tolère pas la différence. Cet autre, ce semblable que l'on voit dans le miroir s'inscrit en quelque sorte dans une différence que l'on ressent comme négation. Si bien qu'un des fondements de la rationalisation du génocide est: « ta peau ou la mienne» ou «ma peau vaut plus que la tienne». MP: Je trouve que c'est une formule très forte qui insiste sur le principe d'inégalité qui régit peut-être le rapport entre les humains : « ma peau vaut plus que la tienne ». Si vous vous laissiez associer sur cette formule-là, qu'en diriez-vous? MB : Je suis heureux de l'invitation qui passe par le mot associer. Je préfère en effet un mode associatif parce que cela m'autorise à dire ce qui vient de façon non préconçue, ce qui me fait penser à un de mes amis et formateurs qui disait: «Je ne produis pas de la pensée comme d'aucuns produisent des sucs gastriques».

«Ta peau ou la mienne», voilà qui nous plonge dans les fondements même de la personnalité, dans le vouloir-être, dans le vouloir-vivre, dans le vouloir-exister. Mais si je veux exister en tant qu'ego, il faut bien que je me singularise, que je me différencie. Par conséquent, l'autre, par sa différence, constitue une menace.

MP : Mais en quoi l'autre est-il une menace?

MB : Celui que je vais tenter d'éradiquer, d'éliminer, ou, si nous nous situons dans un registre plus soft, de critiquer, c'est celui qui m'est le plus proche. 
J'aurais beaucoup de mal à critiquer un Chinois qui vit dans une lointaine province dont je ne connais ni les coutumes ni la pensée. Mais celui qui vit à mes côtés, c'est celui-là que je suis tenté d'éliminer, car son existence est une menace à ma propre existence. Il y a un impérialisme de l'ego que nous connaissons bien en psychanalyse et qui fonctionne sur un plan individuel, mais je pense que cela fait aussi partie de la psychologie collective. La différence est une menace. D'une certaine manière, la différence m'empêche d'être ce que je prétends être ou ce que je veux être. Je n'ai d'autre issue pour être ce que je suis que de tenter d'éliminer l'autre et pour y arriver... tous les coups sont permis, depuis la sournoise critique jusqu'au meurtre ou au génocide. Que signifie la pureté de la race, sinon imposer comme norme universelle un certain mode d'être et de paraitre? Et est-ce que cela ne relève pas de la passion fantasmatique du Un? Effectivement, les génocides s'organisent autour d'un système de pensée qui veut faire du Un. Et ce Un ne tolère pas l'autre.

MP: Alors, lorsque vous dites que nous attendons toujours que Dieu s'explique sur Auschwitz, ce qui constitue une demande impossible, je me demande à quel type d'explication nous pouvons nous attendre. Est-il possible de dépasser le fantasme du Un?

MB : Si j'ai soulevé la question en ces termes, c'est bien pour pointer l'impasse de la conception anthropomorphique de Dieu avec le fantasme de la bonté de Dieu. Quand nous avons une vision anthropomorphique du divin, le problème du mal est absolument intraitable. Le Dieu imaginaire que nous avons construit, peut-être sur des assises symboliques, qui est réputé être bon, ne peut pas rendre compte de cela. C'est la raison pour laquelle j'ai décentré ma vision du divin sur l'Orient, sur le cela, sur la non-dualité qui admet toutes les contradictions. Appartient à ce même réel, à ce cela, l'exquise émotion de l'écoute d'une symphonie de Mozart... et à côté, la vision du chauffard ivre qui écrase la tête de l'enfant que j'aime. Mais l'idée d'un dieu bon est insoutenable, car le réel, tel que nous y sommes confrontés, n’apporte aucune explication, aucune logique, aucune philosophie qui puisse justifier l'horreur dont un dieu bon serait en quelque sorte la caution. C'est pour cette raison que j'éprouve une certaine tendresse pour l'Orient qui arrive à étendre sa notion du divin à la question de la destruction. Le dieu Shiva est un dieu qui inclut dans sa divinité un principe de destruction... et là, dans ces conditions, ça me paraît cohérent.

MP : Comme Kali.

MB : Comme Kali. Étant entendu que Kali est un avatar de Shiva ${ }^{3}$. 
MP: Vous touchez là au cœur de la question du mal en tant qu'elle est liée à celle de l'anthropomorphisation de Dieu. Nous fantasmons un Dieu bon qui viendrait panser les blessures qu'autrement, nous n'arriverions pas à penser. Tant que nous nous situons dans cette perspective-là, ne sommes-nous pas devant une impasse éthique? Il n'y a aucune justification possible, éthique, politique, philosophique, aux grands génocides, aux massacres de masse. Par contre, en nous déplaçant comme vous le proposez du côté du cela, du côté de l'Orient, cela permet au sujet qui a traversé ou qui a été traversé par ces violences extrêmes, en sortant des fantasmes de bonté, de s'étayer sur quelque chose...

MB : Peut-être pourrions-nous faire un court-circuit entre un concept lacanien et un concept qui nous vient du Vedanta ${ }^{4}$ ? Je ferais alors référence au réel, ce qui implique le rapport à l'impossible, donc un réel non duel. MP: Un réel non duel?

MB : La notion de Dieu que nous soutenons s'inscrit dans une perspective dualiste qui nous conduit justement à des impasses logiques et éthiques. Je me souviens d'une entrevue d'Élie Wiesel, écrivain et prix Nobel, qui avait dit "Lorsque je pense au massacre des enfants et des innocents, je ne crois plus à la bonté de Dieu et je n'accorde à Dieu aucune circonstance atténuante. » MP : Il aurait parlé, mais dans son propre vocabulaire, de ce que Jacques Derrida appelle la cruauté sans alibi.

MB : Quelque chose m'est à moi intolérable, un peu en écho à une pensée de Pascal, qui disait à propos de l'esprit religieux: «Dieu, ne nous a pas donné la raison pour que nous renoncions à en faire usage.» Cette notion de Dieu que nous évoquions s'inscrit dans une perspective dualiste avec les impasses qu'on vient d'évoquer, cela met à mal notre raison. Et notre raison nous dit que ce Dieu-là, pure création de l'esprit humain, ne peut pas vraiment exister. Michel Serres dit que «si Dieu était infiniment bon, Il serait en même temps infiniment vulnérable», ce qui, évidemment, est assez peu compatible avec l'idée de sa toute-puissance. Nous ne cessons de nous gargariser de la toute-puissance de Dieu. Or, s'Il était tout-puissant dans le sens où nous les êtres humains l'entendons, comment se fait-il qu'Il puisse tolérer ce que nous jugeons être le mal? Dans le travail que j'ai fait, je dis que Dieu, le Dieu des religions révélées ${ }^{5}$, est une projection de l'idéal du moi. Cependant, les penseurs de l'Ancien Testament ont montré que Dieu était inconcevable, irreprésentable, etcetera. Mais il y a une contradiction à le formuler en ces termes pour ensuite l'anthropomorphiser en lui attribuant toutes sortes de qualités humaines. Cette notion du divin ne me convient pas. 
MP : C'est un véritable défi clinique avec les personnes qui ont traversé certains types de violences extrêmes et s'en remettent entre autres à Dieu de différentes manières, c'est-à-dire à un dieu, qui est précisément ce dieu anthropomorphique dont vous parlez, qui viendrait les sauver et racheter le mal dont ils ont été l'objet, guérissant ainsi leurs blessures et effaçant les coupures, faisant en sorte que les traumatismes subis puissent être, non pas oubliés, mais à tout le moins dépassés. On en appelle à Dieu comme étant Celui qui ne vient peut-être pas effacer, mais en tout cas mettre un baume et permettre de reprendre sa vie en main.

MB : En somme, un Dieu consolateur. Le problème c'est que ça fait plus de deux mille ans que ça dure et nous n'avons vu absolument aucune évolution sensible de l'humanité en dépit des idéaux extrêmement généreux du christianisme. Cela m’a posé question depuis que je suis enfant: Jésus a été qualifié de sauveur, mais nous sommes dans la répétition du pire, sans le moindre changement, puisque l'actualité la plus récente nous prouve que rien n’a bougé. Les bonnes âmes qui disent « Plus jamais ça » en faisant référence à Auschwitz sont dans la méconnaissance que nous sommes toujours là-dedans. Le génocide, pour reprendre ce que nous disions tout à l'heure, c'est le massacre de celui qui nous est proche. Dans ce que j'ai écrit, j'ai désigné les Serbes, c'est un exemple qui me venait notamment parce que j'avais vu des images télévisées qui m'avaient fait beaucoup souffrir... des exécutions en masse. Habituellement, lorsque nous pensons au génocide, nous pensons à la barbarie, à ceux qui sont loin... mais en fait, le barbare est à notre porte. À la limite, le barbare, c'est moi quand je suis sous l'empire d'une idéologie qui pense au Un comme fantasme, à cet Un qui exclut l'altérité de l'autre, cet Un qui exclut la différence.

MP: Comme si le barbare en nous nous menaçait toujours depuis toujours. MB : Oui, et je serais assez freudien pour dire que l'un des fondements du racisme, c'est l'échec de la métaphore paternelle, au sens où le fait de ne pas avoir pu intégrer la différence sexuelle et la différence tout court nous amène au déni de l'altérité de l'autre.

MP : Et donc à la transgression des tabous de l'inceste et du meurtre.

MB : D'un point de vue clinique, on voit bien, par exemple dans la psychose paranoïaque, quand quelque chose a fait échec du côté de la métaphore paternelle, que nous sommes dans une relation en miroir vis-à-vis de l'autre et que celui que l'on accuse, c'est cet autre qui en même temps est soi. C'est ce qu'on ne veut pas voir en soi. Quand le paranoïaque vise la destruction de l'autre, quand il le désigne comme persécuteur, c'est en fait à son propre moi qu'il s'adresse, qu'il s'en prend. 
Toujours sur un mode associatif, cela m'évoque aussi la question du transitivisme chez l'enfant. Le petit garçon rentre chez lui en pleurant et en disant qu'il a été battu alors que c'est lui qui a battu l'autre. Il me semble que, dans ce qui peut fomenter la haine, le désir de détruire, il y a quelque chose qui nous ramène à ce que nous ne voulons pas savoir de notre propre moi.

MP: Je pensais au conflit israélo-palestinien, à cette question du sémitisme, mais cela m'amène à ce dont vous parlez à la fin du troisième chapitre. Vous évoquez alors une remarque faite par Jérôme Garcin dans Le Nouvel Observateur, lorsqu'il écrit "Depuis Gutenberg, il est en effet établi qu'aimer, c'est s'effacer, alors que détester, c'est exister.» Et vous commentez: «Voilà donc exposé avec simplicité le redoutable nouage entre le désir d'être et la haine. Et la haine peut constituer une raison d'exister» $(2012,112)$. J'aimerais vous entendre là-dessus pour clarifier le lien entre cette haine et le "Jamais plus» dont nous discutions à l'instant car il me semble que le nouage entre le désir et la haine se trouve au cœur de chacune de ces violences extrêmes, le cœur intime de cette destructivité presque banalisée aujourd'hui, comme on le voit au cinéma, à la télévision et dans les nouveaux médias. Dans les séries télévisées par exemple, la haine est presque un moteur à partir duquel se promeut l'ego.

MB : Je n'aurais pas grand-chose à ajouter à ce que vous avancez. C'est sur le fondement de la haine que se promeut l'ego qui lui permet de s'isoler dans ses frontières en tant qu'ego, l'ego individuel comme également l'ego collectif, puisque l'identité d'un peuple se construit assez souvent sur l'exclusion de l'autre. En termes topologiques, il y a peut-être quelque chose qui se joue là entre le dedans et le dehors. L'identité d'un peuple se constitue à l'intérieur d'un territoire national délimité par des frontières... et l'autre, celui qui est au-delà de la frontière, celui qui est différent, constitue pour moi une menace et je ne puis me maintenir dans mon identité que par la haine. Si l'autre est comme moi, mon identité ne risque-t-elle pas de se dissoudre? Et puis je voudrais rajouter que si la haine est une raison d'exister, elle est également un mode de jouissance.

MP : C'est un élément extrêmement important. Par exemple, on ne peut pas simplement rapporter le fait que le bourreau exécute un travail au nom d'un État. Il y trouve tout de même quelque chose, de l'ordre de la jouissance de même que dans la répétition traumatique, il y a aussi un élément de jouissance. Dès que l'on procède à ce nouage, dès que l'on établit ce lien entre haine et jouissance, pour des cliniciens, ça paraît évident, mais pour bien d'autres gens, cela peut être choquant - ce qui n'est pas si grave, mais demande explication. 
MB : Oui, c'est extrêmement troublant, d'autant que certaines gens confondent jouissance et plaisir. Le plaisir n'est pas la jouissance. Je pense à un auteur comme Serge Leclaire qui disait: "Le plaisir c'est ce qui dans l'être humain peut faire barrage à la jouissance. » La jouissance, c'est précisément ce qui ne tolère pas de limites et qui est l'expression de la satisfaction de la pulsion. Dans la pulsion, nous avons des pulsions de vie et des pulsions de mort. Maintenant, la haine relève de la pulsion de destruction. Comment peut-on tirer jouissance d'une pulsion de destruction si la jouissance, d'un point de vue spinoziste, c'est ce qui me permet de me maintenir dans mon être? Tout ce qui menace cet être devra être détruit. C’est donc au nom de cette tendance que j'ai en moi à persévérer dans mon être que je veux détruire l'autre qui constitue une menace à ma manière d'être, à ce qui me singularise, à ce qui au fond me permet d'être ce que je suis.

Je ne pense pas que la haine soit possible sans qu'il y ait quelque part une logique de l'enfermement, au sens où ce que je pense être moi, mon ego, ne peut subsister en tant que tel que dans une logique de la clôture. L'autre, mon semblable - je le dis en termes abstraits alors que je cherche à être concret... - peut à tout moment faire irruption dans ma bulle. Pour pouvoir rester dans ma bulle existentielle, soit que je tienne à distance cet autre, soit que je le détruise. Je fais un court-circuit entre être et jouissance. L'être est un être de jouissance. Les Upanishads ${ }^{6}$ disaient : "Les êtres viennent au monde sur le fondement de jouissance, persistent dans l'être par la jouissance et, à leur mort, ils retournent à la pure jouissance. » Un autre texte disait: «La jouissance existait avant tout le reste, avant même le germe de toute pensée.» C'est dire que sans la jouissance serait vaine l'existence de l'univers.

Alors revenons maintenant au niveau individuel. J'associe de façon un peu rapide, le fait de persévérer dans son être et jouir, mais il y a quelque chose de cet ordre-là. On le voit en clinique. Je ne puis me maintenir dans ce que je suis qu'au prix de l'élimination de l'autre et cette élimination de l'autre passe par les formes les plus subtiles, des plus softs jusqu'au plus violentes. La jouissance de la haine, c'est la médisance que nous faisons fonctionner au quotidien. Quel est l'essentiel des conversations des gens? Dire du mal de l'autre. Et ça, c'est une modalité de la haine. Mais si je dis du mal de l'autre, c'est bien parce que j'estime que ma propre position est la seule recevable, la seule valable. J'associe le fonctionnement de la haine au narcissisme. Je ne suis haineux que parce que quelque chose en moi me pousse à penser, à sentir que je vaux plus que l'autre, que mon existence compte plus que celle de l'autre. Quelqu'un qui est désencombré de son narcissisme sera désencombré de toute 
forme de haine. Si le narcissisme existe sur le plan individuel, on le voit aussi fonctionner sur le plan collectif: la recherche d'une identité collective à travers une idéologie est un mode de narcissisme collectif. Mais à partir du moment où je suis ouvert, d'une part, $\mathrm{j}$ 'ai raboté mon narcissisme et d'autre part, en admettant l'existence de l'autre, je ne peux plus être dans la haine.

Toujours sous forme associative, je peux ajouter que la question de l'être, quand elle s'articule à celle de la jouissance, nous renvoie à une vision limitée de l'être. Qu'est-ce qui peut nous sortir de là ? Comment élargir la notion individuelle de l'être? Ça met en cause les frontières à l'intérieur desquelles nous fonctionnons. Je pense que le sujet psychotique, qui a du mal avec les notions de frontières, est en recherche de son être au-delà des frontières. Et ce n'est pas pour rien que bien des délires psychotiques empruntent des voix religieuses ou mystiques.

MP : Cette voix religieuse ou mystique serait conçue chez le psychotique comme un pas au-delà de la frontière.

MB : Oui, nous voilà ramenés au problème de la pensée dualiste à laquelle Lacan a d'ailleurs essayé de faire un sort grâce à la topologie, la bande de Moebius, la bouteille de Klein, lorsqu'il montre comment le dedans et le dehors peuvent faire écho l'un à l'autre. Pour ma part, ce que j'aime bien chez les Orientaux, c'est ce qui fonctionne selon la logique de la non-dualité où une chose peut être ce qu'elle est et en même temps autre chose, où l'Un est en même temps le multiple, où les frontières entre le sujet et l'objet sont subverties. La question de l'éveil qui m'intéresse tant nous renvoie à un mode d'être qui subvertit la relation sujet-objet et nous fait percevoir le Un et la totalité au niveau de la singularité.

MP : En effet, il s'agit d'une problématique extrêmement fondamentale aujourd'hui. La notion même de frontières est maintenant mise à mal, ce qui implique des enjeux politiques importants, comme on le voit par exemple avec la question de la citoyenneté. Qu'est-ce que signifie désormais être citoyen de tel ou tel pays? On voit des gens qui peuvent aujourd'hui posséder plusieurs passeports, parlent plusieurs langues, qui sont dans un passage constant. Combien de migrants ont le désir d'aller dans un ailleurs qui leur apporte quelque chose sur le plan de l'imaginaire, mais aussi sur le plan de la réalité. Cela sans compter les millions de migrants forcés et le vacillement de l'État-Nation soumis à la guerre économique entretenue par une caste d'ultra-riches qui se moque des lois nationales et des frontières. Il y a des enjeux subjectifs, il y a des enjeux collectifs, il y a des enjeux de différentes natures, et quand vous parlez de cette pensée de la non-dualité, de l'éveil, de 
la transgression, de la mise en question des frontières, je me demande si, dans cet horizon, la notion d'éveil peut nous aider à penser.

MB : Il est difficile de l'aborder comme une notion puisqu'elle subvertit la relation même au concept. Dans la mesure où il s'agit de la transgression des frontières, notamment les frontières de l'ego et plus largement certaines frontières territoriales idéologiques, l'éveil constitue une ouverture sur l'universel et pourrait représenter de façon un peu utopique une mutation de la conscience collective. Ça passe d'abord par des « expériences » individuelles, quoique certains hommes que l'on qualifie d'éveillés soient également des éveilleurs. Le nom Bouddha signifie littéralement «l'éveillé». Et l'éveil du Bouddha a donné lieu à un ensemble gigantesque de traditions en Asie du Sud-est, au point que l'on peut parler d'une véritable culture de l'éveil. Le fait qu'il y ait eu en Inde et ailleurs des éveillés n’a empêché ni les guerres, ni les génocides. Alors, c'est pour cela que la question de l'éveil pour moi demeure à la fois un idéal, une utopie, mais aussi un espoir. L'espoir d'une mutation de la conscience humaine, peut-être dans quelques milliers d'années, quand nous aurons compris que du côté de l'ego et du sujet conçu à l'occidentale, nous sommes dans des impasses. Tant que nous sommes à la recherche de la jouissance, nous sommes dans des impasses. Le sujet éveillé ne recherche pas la jouissance, il accueille ce qui peut se donner comme bonheur et plaisir sans se lancer dans la recherche effrénée d'un objet de jouissance qui va en quelque sorte l'aliéner. Notre société contemporaine est fondée sur une idéologie de la jouissance. C'est ce que nous recherchons à travers le capitalisme, la production de nouveaux objets, et nous ne semblons pas avoir encore pris la mesure du fait que le bonheur d'être ne se situe pas dans la relation à un objet. Nous sommes dans une confusion idéologique majeure entretenue par la publicité, laissant croire que le bonheur est dans l'avoir plutôt que dans l'être. Si nous étions un peu attentifs à ce qui se passe chez les dictateurs, chez tous ces potentats qui ont tout plus le reste, eh bien, nous pourrions constater qu'ils sont demeurés d'éternels insatisfaits. Kadhafi possédait dans son palais plus de 200 voitures de collection, il avait toutes les femmes qu'il voulait, et pourtant, il éprouvait le besoin de violer des petites filles. Avoir, avoir, avoir! C'est un processus sans fin. La recherche de jouissance est vouée à l'impasse. Le sujet éveillé est un non-sujet qui rompt radicalement avec la recherche de la jouissance, car le fait d'être, simplement d'être, est une plénitude qui n'appelle rien d'autre qu'elle-même et ce, même si au niveau du réel, le sujet éveillé est confronté à la souffrance ordinaire. 
MP : Michel Brun, merci de m'avoir accordé cette entrevue qui ouvre sur des perspectives souvent peu considérées dans la psychanalyse actuelle.

\section{Notes}

1. Cette entrevue a été réalisée à Paris, en 2013.

2. Michel-Yvon Brun, Dieu, encore? Jalons pour une théologie négative contemporaine, Collection Voix psychanalytiques, Montréal, Liber, 2012.

3. Kali est une «divinité redoutable du panthéon hindouiste, épouse de Shiva, déesse de la Mort» (Larousse).

4. Doctrine philosophique qui interprète les vedas — livres sacrés — hindous.

5. Religions dont le savoir a été révélé aux Hommes par Dieu lui-même ou par ses intermédiaires (des prophètes, par exemple).

6. «Textes sacrés hindous considérés comme révélés et qui datent de la fin de la période védique (entre 700 et 300 av. J.-C.)» (Larousse). 